\title{
Ultrafast Exciton Decay in PbS Quantum Dots through Simultaneous Electron and Hole Recombination with a Surface-Localized Ion Pair
}

\author{
KedyEdme, Stephanie Bettis Homan, Alexander B. Nepomnyashchii, and Emily A. Weiss * \\ Department of Chemistry, Northwestern University, 2145 Sheridan Rd., Evanston, IL 60208- \\ 3113
}

\section{RECEIVED DATE (automatically inserted by publisher);}

*Email: e-weiss@northwestern.edu

\begin{abstract}
This paper describes the ultrafast decay of the band-edge exciton in PbS quantum dots (QDs) through simultaneous recombination of the excitonic hole and electron with the surface localized ion pair formed upon adsorption of tetracyanoquinodimethane (TCNQ). Each PbS QD (R=1.8 $\mathrm{nm}$ ) spontaneously reduces up to 17 TCNQ molecules upon adsorption of the TCNQ molecule to a sulfur on the QD surface. The photoluminescence of the PbS QDs is quenched in the presence of the reduced TCNQ species through ultrafast ( $\leq 15 \mathrm{ps})$ non-radiative decay of the exciton; the rate constant for the decay process increases approximately linearly with the number of adsorbed, reduced TCNQ molecules. Near-infrared and mid-infrared transient absorption show that this decay occurs through simultaneous transfer of the excitonic electron and hole, and is assigned to a four-carrier, concerted charge recombination mechanism based on the observations that (i) the PL of the QDs recovers when spontaneously reduced $\mathrm{TCNQ}^{1-}$ desorbs from the QD surface upon addition of salt, and (ii) the PL of the QDs is preserved when another spontaneous oxidant, ferrocinium, which cannot participate in charge transfer in its reduced state, is substituted for TCNQ.
\end{abstract}




\section{INTRODUCTION}

This paper describes the ultrafast $(\leq 15-\mathrm{ps})$ decay of the lowest-energy exciton in $\mathrm{PbS}$ quantum dots (QDs) through simultaneous decay of the electron and hole in the presence of surface-adsorbed tetracyanoquinodimethane (TCNQ), Scheme 1. Upon adsorption to the QD surface, TCNQ spontaneously accepts electron density from a surface sulfur to form either a localized ion pair (oxidized sulfur- $\mathrm{TCNQ}^{1-}$ ) or charge transfer $(\mathrm{CT})$ complex (oxidized sulfur$\mathrm{TCNQ}^{\mathrm{x}-}, 1<\mathrm{x}<2$ ).[1] A single PbS QD forms ground state ion pairs and CT complexes with multiple (up to 17 for these QDs) adsorbed TCNQ molecules simultaneously. Upon photoexcitation of the QD within these complexes, we observe non-radiative, picosecondtimescale recombination of the exciton; the rate constant for this recombination increases approximately linearly with an increasing number of oxidized sulfur-TCNQ ${ }^{\mathrm{x}}$ pairs per QD. Desorption of $\mathrm{TCNQ}^{1-}$ from the surface of the QD (by adding a counterion), or substitution of TCNQ with another spontaneous oxidant whose reduced

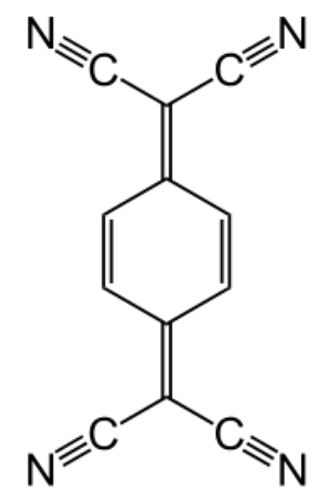

Scheme 1.The structure of TCNQ. form is not redox active with the QD, recovers the PL. These data strongly suggest that excitonic decay occurs via simultaneous charge recombination of the excitonic electron and hole with the electron and hole of the surface-localized ion pair or CT complex. This proposed charge transfer mechanism is a Dexter-type energy transfer,[2] the typical mechanism for triplet-triplet energy transfer,[3,4] where the excitonic electron and hole are recombining with a charge-separated state spread over two distinct sites, rather than to a single redox or chromophoric center. A similar concerted mechanism was observed for the ultrafast transfer of both an electron and hole from the charge-transfer-type excited state of a molecular chromophore, 4-aminonaphthalene-1,8-imide, to separate covalently linked redox partners.[5] The excited state of this chromophore, which has $\sim 70 \%$ charge transfer character, is an organic analog of the exciton in a colloidal QD.

Identifying and eliminating the recombination centers for QD excited states is important for the application of QDs as photosensitizers in heterogeneous or solution-phase photocatalytic systems. QDs have size-tunable absorption spectra that span the ultraviolet, visible, and near- 
infrared regions of the solar spectrum,[6] and highly degenerate band-edge orbitals, which allows them to accommodate and transfer multiple redox equivalents to molecular catalysts (or directly to a catalytic substrate) upon photoexcitation.[7,8] During a sequential multi-electron transfer process, the QD may be photoexcited in the presence of a pre-formed ion pair, where one or both radical ions are located on a proximate redox center, in the core of the QD, or on the surface of the QD. Here we show that charge recombination of the excitonic carriers with these ions is potentially competitive with subsequent electron transfer steps, even if those electron transfers are on the picosecond timescale.

\section{EXPERIMENTAL METHODS}

Synthesis and Purification of PbS Quantum Dots. We synthesized PbS QDs $(\mathrm{R}=1.8 \mathrm{~nm})$ using a procedure adapted from that reported by Hines and Scholes.[9] We added $2 \mathrm{~mL}$ of oleic acid (OA), $18 \mathrm{~mL}$ of 1-octadecene (ODE), and $0.36 \mathrm{~g}$ of $\mathrm{PbO}$ in a three-neck 50-mL roundbottom flask, and heated the mixture to $150{ }^{\circ} \mathrm{C}$ with stirring under nitrogen flow for 30 minutes until the solution became clear and colorless. After cooling the solution to $110{ }^{\circ} \mathrm{C}$, we injected 8 $\mathrm{mL}$ of a hexamethyldisilathiane (TMS) ${ }_{2} \mathrm{~S}$ solution, initially prepared by adding $0.17 \mathrm{~mL}$ of $(\mathrm{TMS})_{2} \mathrm{~S}$ in $8 \mathrm{~mL}$ of $\mathrm{ODE}$, into the round-bottom flask. The $\mathrm{PbO}$ reaction mixture went from colorless to dark brown $\sim 3$ seconds after injection of $(\mathrm{TMS})_{2} \mathrm{~S}$. We allowed the QDs to grow for 10 minutes, and then submerged the flask in an ice bath until the reaction mixture reached room temperature.

We purified the QDs by equally dividing the room-temperature $\mathrm{PbS}$ QDs dispersions into two falcon tubes to which we added acetone in a 1:1 ratio by volume. The tubes were centrifuged at $3500 \mathrm{rpm}$ for 5 minutes, which produced a brown pellet and a supernatant containing excess ODE. After removing the acetone, we redispersed the pellets in a minimal amount of hexanes, addedmethanol in 2:1 excess (by volume), and centrifuged the tubes at $3500 \mathrm{rpm}$ for 5 minutes. The methanol wash also resulted in a brown pellet and a supernatant containing excess ligands and small nanocrystalline clusters. We repeated the methanol wash a second time, centrifuged the mixture, dried the resulting pellets over nitrogen, and redispersed them in chloroform to make stock solutions of PbS QDs.

Bulk Electrolysis.We carried out bulk electrolysis of TCNQ in anhydrous dichloromethane. The samples were prepared in a nitrogen-purged glovebox. We used a three-electrode 
configuration, with a platinum mesh as the working electrode, a platinum counter electrode, and a silver wire as the reference electrode. A porous frit was used to separate the electrodes. We used a CH660 potentiostat( $\mathrm{CH}$ Instruments)to apply a constant potential of $-0.3 \mathrm{~V}$ vs. ferrocene for 40 minutes to a solution of $0.54 \mathrm{mM}$ TCNQ with $0.1 \mathrm{M} \mathrm{TBAPF} 6$ as the supporting electrolyte.

Fourier-transform Infrared (FTIR) Spectroscopy. We performed FTIR spectroscopy on a Thermo-Electron Nicolet NEXUS 870 FTIR in a 0.52-mm path length $\mathrm{KCl}$ cell. We performed measurements on samples in $\mathrm{CHCl}_{3}$ containing $17.8 \mu \mathrm{M} \mathrm{PbS}$ QDs in the presence of $0,0.30$, $0.62,1.2,3.7,6.2,8.6,12.4,30.9,37.1,43.3$ and 49.4 equivalents of TCNQ.

Near-Infrared Transient Absorption (TA) Measurements.Samples for visible-to-NIR TA were prepared at a QDconcentration of $17.8 \mu \mathrm{M}$ and placed in a 2-mm path-length cuvette with quartz windows.The TA system is described in detail elsewhere.[10]For measurements of picosecond-timescale dynamics, we split the output of a commercial amplified Ti-sapphire (Soltice, $1 \mathrm{kHz}, 350 \mathrm{fs}$ ) with output energy of $2.3 \mathrm{~mJ}$ into an optical parametric amplifier (OPA) (TOPAS-C, Light Conversion), to produce a pump wavelength of $900 \mathrm{~nm}$, and into a TA spectrometer (Helios, Ultrafast systems) to produce a white light continuum, which serves as the broadband probe. The pump and the probe were spatially and temporally overlapped on the sample in a 2-mm quartz cuvette.

We measured the microsecond dynamics of the samples in a 2-mm quartz cuvette using the same pump and a commercial EOS spectrometer, which is also described in detail elsewhere.[10]

Mid-Infrared TA.Samples for mid-IR TA were prepared at a concentration of $17.8 \mu \mathrm{M}$ and placed in a $390 \mu \mathrm{m}$ path length IR cell with $\mathrm{CaF}_{2}$ windows. Our ultrafast mid-IR TA set-up splits the output of a commercial amplified Ti:Sapphire laser (Spitfire, 1 kHz, $100 \mathrm{fs}$, Spectra Physics), and guides $50 \%$ to an optical parametric amplifier (OPA) (TOPAS-C, Light Conversion), which produces the pump wavelength $(900 \mathrm{~nm})$ for sample excitation, and $50 \%$ to a second OPA (TOPAS, Light Conversion) to generate the probe pulse. The probe pulses at $4 \mu \mathrm{m}$ and $5 \mu \mathrm{m}$ are the difference frequencies of the signal and idler outputs of the second OPA. The signal is set to $1333 \mathrm{~nm}$ and the idler is set to $2000 \mathrm{~nm}$ for the $4 \mu \mathrm{m}$ output. The signal is set to $1379 \mathrm{~nm}$ and the idler is set to $1905 \mathrm{~nm}$ for the $5 \mu \mathrm{m}$ output. The differential absorption spectrum $(\Delta \mathrm{A})$ was obtained through active background subtraction of the ground state spectrum by chopping the pump pulse at $500 \mathrm{~Hz}$. The pump pulse is focused to $390 \mu \mathrm{m}$ spot size, and overlapped with the 
probe in the sample cell. The transmitted probe pulse is focused into a monochromator that disperses it onto a multichannel mercury cadmium telluride (MCT) detector array (Infrared Systems).

${ }^{1}$ H Nuclear Magnetic Resonance Spectroscopy (NMR). We performed ${ }^{1} \mathrm{H}$ NMR experiments on an Agilent DD2 $600 \mathrm{MHz}$ spectrometer. The samples consisted of $17.8 \mu \mathrm{M} \mathrm{PbS}$ QDs in the absence and presence of $50 \mu \mathrm{Mferrociniumtetrafluoroborate}\left(\mathrm{Fc}^{+} / \mathrm{BF}_{4}^{-}\right)$in $\mathrm{CDCl}_{3}$.

\section{RESULTS AND DISCUSSION}

Spontaneous reduction of TCNQ by PbS QDs.Figure 1A (inset) shows the ground state absorption spectrum of the $17.8 \mu \mathrm{MPbS}$ QDs used in this study.[11] The spectrum includes a first excitonic peak centered at $985 \mathrm{~nm}$ and a continuum that spans the entire visible region. Analysis of TEM images of the QDs yields an average radius of $1.8 \pm 0.3 \mathrm{~nm}$ (see the Supporting Information, Figure S1B,C).

Figure 1A also shows absorption spectra of reduced TCNQ produced by bulk electrolysis in the presence of $\mathrm{TBAPF}_{6}\left(\right.$ "TBA ${ }^{+} / \mathrm{TCNQ}^{1-» "}$, red), as described in the Experimental Methods section, and of a dispersion of PbS QDs with 2.5 added equivalents of TCNQ (black) after subtraction of the spectrum of QDs alone. The shape of the spectrum of TCNQ in the presence of QDs is very similar to the spectrum of electrochemically reduced TCNQ, which contains characteristic $\mathrm{TCNQ}^{1-}$ absorption features at 686,749 and $850 \mathrm{~nm} ;[1,12,13]$ these spectra indicate that spontaneous electron transfer from PbS QDs to TCNQ forms a stable $\mathrm{QD}^{1+} / \mathrm{TCNQ}^{1-}$ complex. From the intensity of the signal corresponding to neutral TCNQ (centered at $400 \mathrm{~nm}$, not shown), we calculate that the mixture of QDs with TCNQ contains $2.4 \mu$ Mof neutral TCNQ in solution, and therefore $(4.2-2.4=) 1.8 \mu \mathrm{M}$ of reduced TCNQ in the sample (there is no signal from $\left.\mathrm{TCNQ}^{2-}\right)$. Using the observed absorbance of reduced TCNQ at $842 \mathrm{~nm}(0.026$ a.u), we calculate the extinction coefficient of the surface-adsorbed, reduced TCNQ to be $14,000 \mathrm{~cm}^{-1} \mathrm{M}^{-}$ ${ }^{1}$, which is a factor of 3 smaller than the published value of $43,300 \mathrm{~cm}^{-1} \mathrm{M}^{-1}$ in $\mathrm{CH}_{2} \mathrm{Cl}_{2}$.[12]For comparison, we have scaled the spectrum of pre-electrolyzed $\mathrm{TCNQ}^{1-}$ such that the two spectra correspond to the same concentrations of reduced TCNQ; we see, again, that the signal from free $\mathrm{TCNQ}^{1-}$ in solution is a factor of 3 larger than that of the TCNQ reduced in situ.

The ground state absorption of the QD does not bleach upon addition of TCNQ (see the Supporting Information, Figure S1A). The lack of a ground state bleach and the absence of the 
characteristic intraband absorptions[14] of an excitonic hole in the mid-IR spectrum of QDTCNQ complexes (see the Supporting Information, Figure S2) are consistent with our previous conclusion[1] that TCNQ is reduced by the surface sulfurs to which they adsorb, and not by the QD core. In that previous study[1], we supported our assertion that the oxidized species is at the surface of the QD (as opposed to a freely diffusing molecular species) by showing that the spinlattice $\left(\mathrm{T}_{1}\right)$ relaxation times of protons on the oleate ligands bound to the QDs are accelerated by the reduction of TCNQ in mixtures with the QD, due to the paramagnetism of $\mathrm{TCNQ}^{1-}$, of the surface hole, or both. The degree to which $T_{1}$ is accelerated is precisely proportional to the amount of reduced TCNQ in the system (as measured by FTIR). Regardless of the origin of the paramagnetism (i.e., whether it is all due to $\mathrm{TCNQ}^{1-}$, or whether some originates from the hole of the reductant species), the paramagnetic species must be localized on the surface of the QD in order to see this acceleration. If the hole were localized on a free molecular species that could diffuse away from the QD, then $\mathrm{TCNQ}^{1-}$ would also diffuse away because these ions would not exist unpaired in organic solvent, and we would see no acceleration of $T_{1}$. 

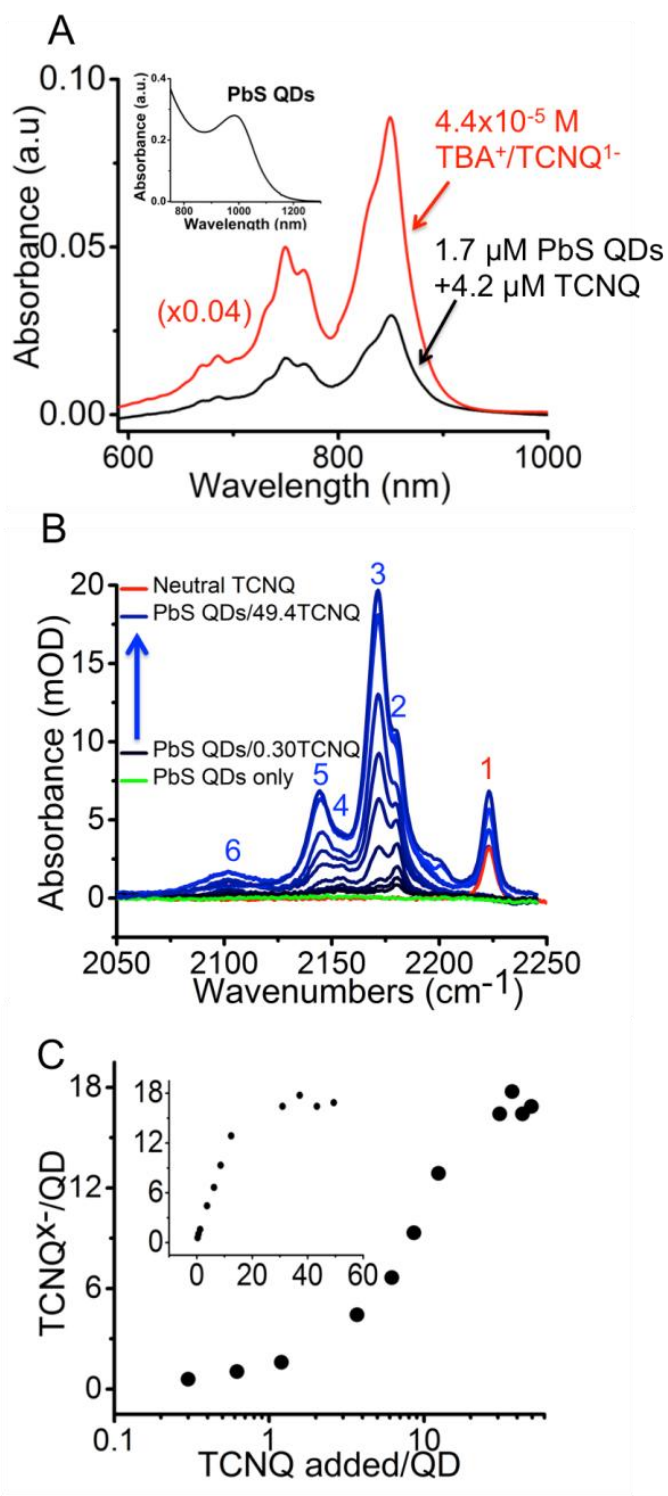

Figure 1.A) Absorption spectra of reduced TCNQ produced by bulk electrolysis in the presence of $\mathrm{TBAPF}_{6}$ ("TBA ${ }^{+} / \mathrm{TCNQ}^{1-\text { ", }}$, red) and of a mixture of $\mathrm{PbS} \mathrm{QDs}(\mathrm{R}=1.8 \mathrm{~nm}$ ) with 2.5 equivalents of TCNQ (black) after subtraction of the QD-only spectrum, which peaks at $985 \mathrm{~nm}$ (inset). B)The nitrile stretching region within FTIR spectra of TCNQ with no added QDs (red), and of $17.8 \mu \mathrm{M} \mathrm{PbS}$ QDs with increasing concentrations of added TCNQ (black to blue). The peaks correspond to C-N stretching modes for neutral TCNQ (1), for TCNQ ${ }^{1-}(\mathbf{2}$ and $\mathbf{4})$ and for $\mathrm{TCNQ}^{\mathrm{x}-}$ where $1<\mathrm{x}<2 \quad\left(\mathbf{3}, \mathbf{5}\right.$ and 6). C)Plot of the number of $\mathrm{TCNQ}^{\mathrm{x}-}$ formed in situper PbS QD $(\mathrm{R}=1.8 \mathrm{~nm}$, [QDs] $=17.8 \mu \mathrm{M})$ vs. the number of equivalents of added TCNQ per QD. We determined the number of $\mathrm{TCNQ}^{\mathrm{X}} / \mathrm{QD}$ using the combined signal intensity of peaks $\mathbf{2}$ and $\mathbf{3}$ inpanel B.Inset: The same plot as $\mathrm{C}$, but plotted on a linear scale to show more clearly that the number of reducing sites on the QD surfaces for TCNQ saturates at approximately 17 per QD. 
Figure 1B shows the nitrile stretching region of the FTIR spectra of neutral TCNQ (red, peak 1), and of reduced TCNQ generated within samples containing a mixture of 17.8 $\mu \mathrm{M}$ PbS QDs with varying concentrations of TCNQ. The spectrum of $\mathrm{TCNQ}^{\mathrm{x}-}$ in this region, where $1 \leq \mathrm{x}<2$, is known to contain a pair of peaks separated by $\sim 25 \mathrm{~cm}^{-1}$ with energies that decrease as $\mathrm{x}$ increases if the charge is spread symmetrically over the four nitrile groups.[15] In our samples, when the molar ratio TCNQ:QD is $<3.7: 1$, the dominant pair of peaks in the spectra in Figure 1B are those at $2179 \mathrm{~cm}^{-1}$ (“2”) and $2154 \mathrm{~cm}^{-1}$ (“4”). Based on the FTIR spectrum of mixtures of decamethylferrocene (DFe) and TCNQ, where DFe spontaneously reduces TCNQ to TCNQ ${ }^{1-}$, we determined that the peaks (“2”) and (“4”) correspond to TCNQ ${ }^{1-}$ (see Supporting Information, Figure S3). When the molar ratio TCNQ:QD $\geq 3.7: 1$, the pair of peaks at $2170 \mathrm{~cm}^{-1}$ (“3”) and $2145 \mathrm{~cm}^{-1}$ (“5”)become the dominant pair. The growth of peaks " 3 " and "5" is correlated with the growth of a peak at $2100 \mathrm{~cm}^{-1}$ (“6”).[15]These peaks correspond to TCNQ ${ }^{\mathrm{x}-}$, where $1<x<2$. The different charge states of TCNQ correspond to different adsorption geometries. $\mathrm{TCNQ}^{1-}$ exists within an electrostatically bound ion pair of TCNQ with surface sulfur, while $\mathrm{TCNQ}^{\mathrm{x}-}$, where $1<\mathrm{x}<2$, exists within strongly coupled CT complexes of TCNQ with surface sulfur, where charge is shared between the donor and acceptor. We discuss these geometries in detail elsewhere.[15]At molar ratios TCNQ:QD > 30.9:1, we observe contributions from neutral TCNQ (peak 1) in the FTIR spectra of the mixtures, due to saturation of available adsorption sites on the QD surfaces and the presence of a steady-state population of freely diffusing TCNQ.

Using the intensities of peaks $\mathbf{2}$ and3(representative of the two charge states of TCNQ), in these FTIR spectra, we quantify the number of spontaneously reduced TCNQ molecules per QD for each concentration of added TCNQ. We first calculate an effective extinction coefficient for the reduced TCNQ species by summing the areas of peaks $\mathbf{2}$ and $\mathbf{3}$ within spectra of samples containing no neutral TCNQ - that is, samples where all added TCNQ is reduced by the QDs and plotting this total area vs. the concentration of added TCNQ. In these samples, we assume that the concentration of added TCNQ equals the concentration of reduced TCNQ. This procedure yields $\varepsilon_{\mathrm{eff}}=2.4 \times 10^{4} \mathrm{M}^{-1} \mathrm{~cm}^{-2}$ (see Supporting Information, Figure S4). We then use this extinction coefficient to calculate the concentration of all species of reduced TCNQ in every sample. Figure 1 Cshows that the number of $\mathrm{TCNQ}^{\mathrm{x}-}$ per $\mathrm{QD}$ increases with increasing 
concentration of added TCNQ, with a maximum of approximately $17 \mathrm{TCNQ}^{\mathrm{x}-}$ molecules per QD in the sample.

TCNQ quenches the photoluminescence of $\mathrm{PbS}$ QDs. Figure $2 \mathrm{~A}$ shows a decrease in the photoluminescence (PL) intensity of the QDs as the concentration of added TCNQ increases. The absorption cross-section of the QDs at the band-edge wavelength does not change upon addition of TCNQ, and we do not observe any defect state emission at wavelengths longer than the band-edge wavelength, so the decrease in the PL intensity of the samples is due to an additional non-radiative relaxation pathway for the photogenerated exciton in the QDs due to the adsorption of TCNQ. The Supporting Information details how the PL measurements were performed.

Figure 2B shows a plot of "PL/PL,", the fraction of originally emissive QDs in the sample that still undergo radiative decay after the addition of TCNQ, vs. the concentration of bound $\mathrm{TCNQ}^{\mathrm{x}-}$. At a molar ratio $\mathrm{TCNQ}^{\mathrm{X}-}: \mathrm{QD}$ of 8.6:1, the PL of the QD sample is quenched by more than $99 \%$.

Surface-adsorbed TCNQ ${ }^{\mathrm{x}-}$ induces an ultrafast decay of the QD excited state. In order to determine the mechanism by which adsorbed $\mathrm{TCNQ}^{\mathrm{x}-}$ quenches the PL of QDs, we studied the decay dynamics of the excitonic state of the QDs in the presence of TCNQ with transient absorption (TA) spectroscopy in the near-IR and mid-IR. Figure 3A shows a kinetic trace extracted from the TA spectrum of $17.8 \mu \mathrm{M}$ PbS QDs in the absenceof TCNQ at $1000 \mathrm{~nm}$ (corresponding to the ground state bleach, orange); as expected, it does not recover on the picosecond timescale. We show in the Supporting Information, Figure S5, that the exciton lifetime of the QDs in the absence of TCNQ is $2.3 \mu$ s. Figure 3A alsoshows that increasing the number of bound $\mathrm{TCNQ}^{\mathrm{x}-}$ per QD (for 3.7, 6.2, 8.6, and 12.4 boundTCNQ ${ }^{\mathrm{x}-}$ per QD) induces an ultrafast recovery of the ground state bleach. We fit the kinetic traces to a sum of exponential functions convoluted with an instrument response function (IRF). 

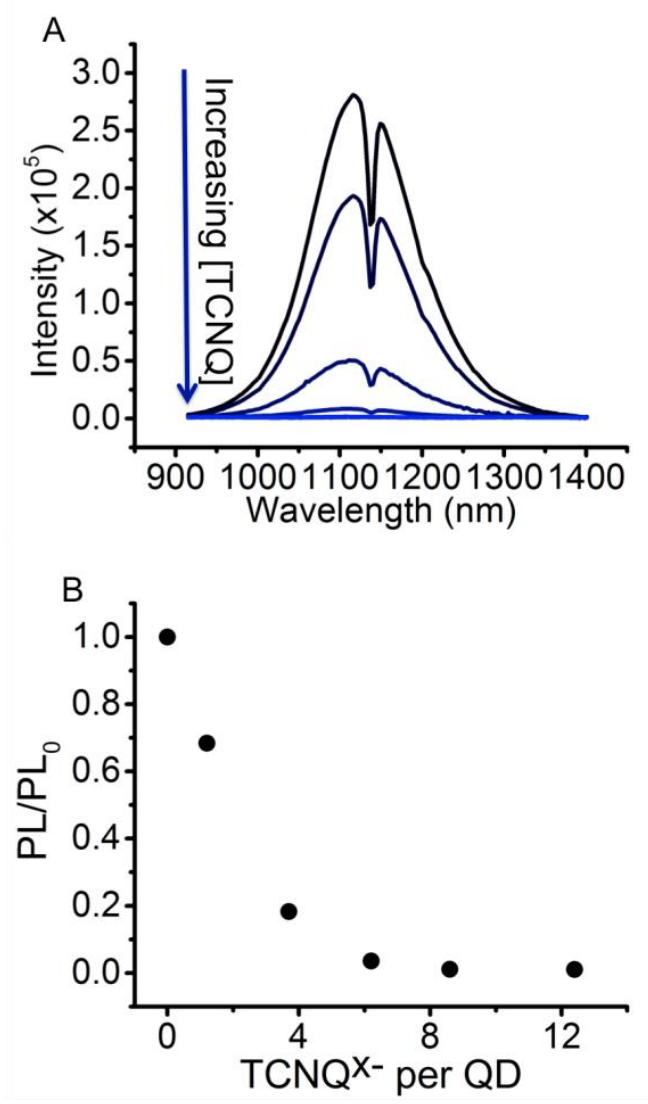

Figure 2. A) The PL spectra of $17.8 \mu \mathrm{M} \mathrm{PbS}$ QDs $(\mathrm{R}=1.8 \mathrm{~nm})$ with increasing concentrations of added TCNQ in $\mathrm{CHCl}_{3}$, with a maximum ratio TCNQ:QDof12.4:1. The negative feature at 1140 $\mathrm{nm}$ is due to absorption of the solvent. B) Fraction of originally emissive QDs that remain emissive $\left(\mathrm{PL}_{\mathrm{PL}}\right)$ upon adsorption of various numbers of TCNQ molecules per $\mathrm{QD}$. 

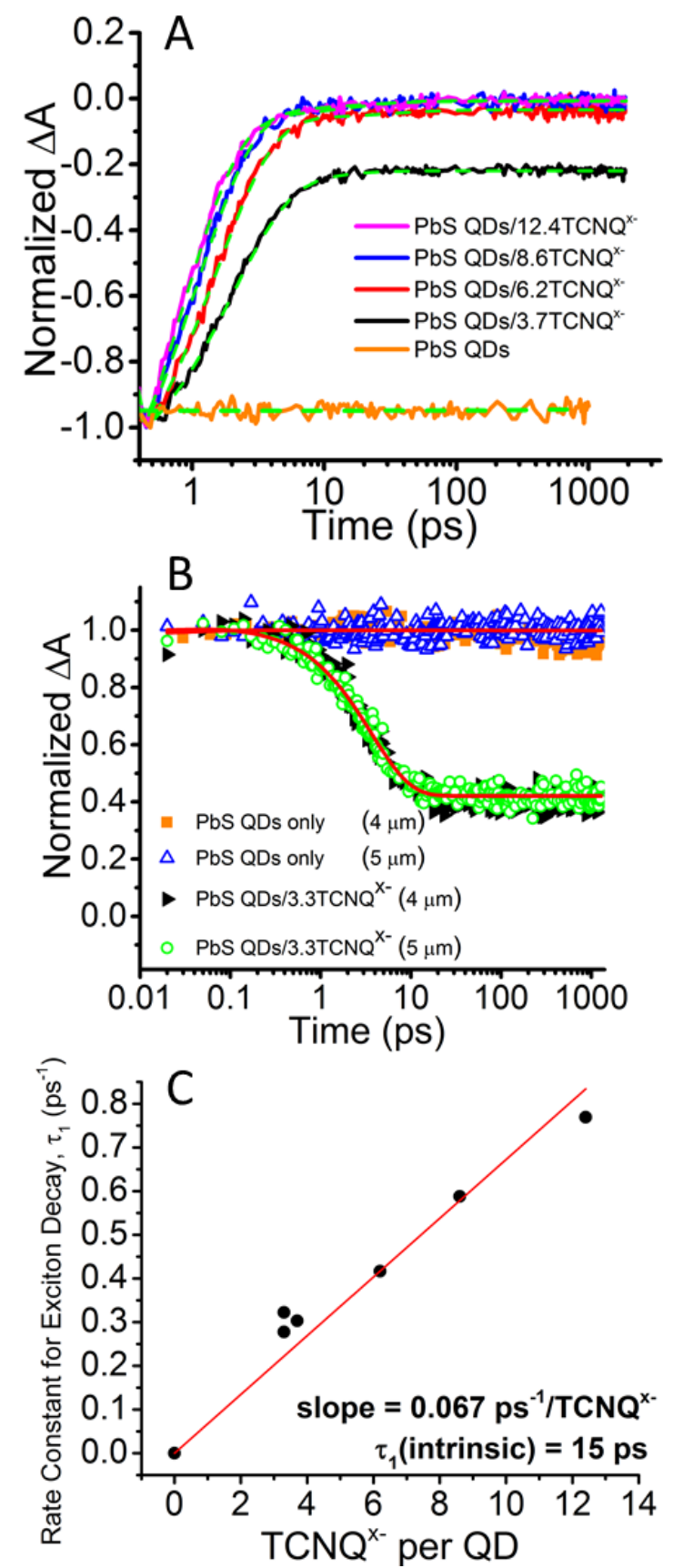

Figure 3.A)Kinetic traces extracted at $1000 \mathrm{~nm}$ from the $\mathrm{TA}$ spectra of $17.8 \mu \mathrm{M} \mathrm{PbS}$ QDs $(\mathrm{R}=$ $\left.1.8 \_n m\right)$ in the presence various equivalents of bound $\mathrm{TCNQ}^{\mathrm{x}}$, in $\mathrm{CHCl}_{3}$, fit to a sum of simple exponential functions convoluted with an IRF (green dashed lines). Table 1 contains the fitting parameters for these traces. B) Normalized kinetic traces extracted at 4 and $5 \mu \mathrm{m}$ from the midIR TA spectra ofsamples of $\mathrm{PbS} \mathrm{QDs}(\mathrm{R}=1.8 \mathrm{~nm})$ in the absence of TCNQ(orange squares and blue opentriangles), and in the presence of 3.3 bound $\mathrm{TCNQ}^{\mathrm{X}}$ - per QD (black triangles and green circles). The 4- $\mu \mathrm{m}$ kinetic trace overlays the 5- $\mu \mathrm{m}$ trace, showing that the electron and hole are annihilated in one concerted step.C) Plot of the measured rate constant for simultaneous electron/hole decay in the presence of adsorbed $\operatorname{TCNQ}^{\mathrm{x}-}\left(1 / \tau_{1}\right)$ in $\mathrm{ps}^{-1}$, vs. the number of 
adsorbed TCNQ ${ }^{\mathrm{x}-}$ per QD, determined from FTIR spectra. The slope of the linear fit to this data yields an "intrinsic" (per-adsorbed-TCNQ ${ }^{\mathrm{x}-}$ ) time constant for excitonic decay of $15 \mathrm{ps}$.

The kinetic trace for the sample with 3.7 boundTCNQ ${ }^{\mathrm{x}-}$ per QD fits to a sum of three exponential components: $\tau_{1}=3.3 \mathrm{ps}$ ( $74 \%$ of the recovery of the bleach signal), $\tau_{2}=16 \mathrm{ps}(5 \%$ of the recovery of the bleach signal), and $\tau_{3}$, which corresponds to the offset of the bleach signal from zero amplitude at long times. We fix $\tau_{3}$ to $2.3 \mu$ s to account for recombination of excitons within QDs that do not participate in the ultrafast exciton decay. The Supporting Information, Figure S6, shows that inclusion of the smallest-amplitude component, $\tau_{2}$, is necessary to achieve a symmetric scattering of residuals around the zero line, and therefore an adequate fit. Mid-IR TA experiments, described below, which differentiate electron dynamics from hole dynamics, suggest that the $\tau_{2}$ component is due to a small fraction of QDs participating in an electron trapping mechanism (see the Supporting Information, Figure S7); we will not discuss this component further. The value of $\tau_{1}$ shortens to $2.4 \mathrm{ps}$ for the sample with $6.2 \mathrm{TCNQ}^{\mathrm{X}-}$, to $1.7 \mathrm{ps}$ for $8.6 \mathrm{TCNQ}^{\mathrm{x}-} / \mathrm{QD}$, and to $1.3 \mathrm{ps}$ for $12.4 \mathrm{TCNQ}^{\mathrm{x}-} / \mathrm{QD}$. Traces for the samples with 8.6 and 12.4TCNQ ${ }^{\mathrm{x}} / \mathrm{QD}$ do not require the $\tau_{3}$ component to achieve an adequate fit since the PL of these samples is quenched by more than $99 \%$ and the bleach signal recovers to zero on the picosecond timescale. Table 1 summarizes the values of the fitting parameters for the bleach dynamics of each sample.

It appears from these experiments that, for QDs with adsorbed TCNQ, both excitonic carriers (electron and hole) decay with the same ultrafast lifetime $\left(\tau_{1}\right)$, and that this lifetime shortens as the number of adsorbed TCNQ per QD increases. One possibility is that one of the charge carriers is decaying on a timescale that is faster than the instrument response. In this scenario, however, the amplitude of the bleach within the TA spectrum of a sample of PbS QDs in the presence of TCNQ would be less than that of a sample without TCNQ at the earliestdelay times that we can monitor, because the bleach would have already partially recovered due to removal of one the excitonic carriers from the band-edge. In the Supporting Information, Figure S8, we show that the initial amplitudes of the ground state bleach in the TA spectra of samples consisting of $17.8 \mu \mathrm{M}$ PbS with 3.7 bound $\mathrm{TCNQ}^{\mathrm{x}-}$ and without added TCNQ arenearly identical at a delay time of $600 \mathrm{fs}$.We can therefore conclude that neither of the charge carriers decays on a timescale faster than the instrument response. 
Table 1.Fitting Parameters for the near-IR and Mid-IR TA Decay Traces for PbS QDs and Mixtures of PbS QDs and TCNQ.

\begin{tabular}{|c|c|c|c|c|}
\hline Sample & $\begin{array}{l}\text { Probe } \\
\text { Wavelength }\end{array}$ & $\tau_{1}\left(\mathbf{A}_{1}\right)$ & $\tau_{2}\left(\mathbf{A}_{2}\right)$ & $\tau_{3}\left(\mathbf{A}_{3}\right)$ \\
\hline PbS QDs & $1000 \mathrm{~nm}$ & not present & not present & $2.3 \mu \mathrm{s}$ \\
\hline $\mathrm{PbS}$ QDs/3.7 bound $\mathrm{TCNQ}^{\mathrm{x}-}$ & $1000 \mathrm{~nm}$ & $3.3 \mathrm{ps}(0.74)$ & $16 \mathrm{ps}(0.05)$ & $2.3 \mu \mathrm{s}(0.21)^{a}$ \\
\hline $\mathrm{PbS}$ QDs/3.3 bound $\mathrm{TCNQ}^{\mathrm{x}-}$ & $4 \mu \mathrm{m}$ & $3.1 \mathrm{ps}(0.55)$ & $8.9 \mathrm{ps}(0.09)$ & $2.3 \mu \mathrm{s}(0.36)^{a}$ \\
\hline $\mathrm{PbS}$ QDs/3.3 bound $\mathrm{TCNQ}^{\mathrm{x}-}$ & $5 \mu \mathrm{m}$ & $3.6 \mathrm{ps}(0.60)$ & not present & $2.3 \mu \mathrm{s}(0.40)^{a}$ \\
\hline $\mathrm{PbS}$ QDs/6.2 bound $\mathrm{TCNQ}^{\mathrm{x}-}$ & $1000 \mathrm{~nm}$ & $2.4 \mathrm{ps}(0.93)$ & $16 \mathrm{ps}(0.04)$ & $2.3 \mu \mathrm{s}(0.03)^{a}$ \\
\hline $\mathrm{PbS}$ QDs/8.6 bound $\mathrm{TCNQ}^{\mathrm{x}-}$ & $1000 \mathrm{~nm}$ & $1.7 \mathrm{ps}(0.95)$ & $16 \mathrm{ps}(0.05)$ & not present \\
\hline $\begin{array}{lll}\mathrm{PbS} & \text { QDs/12.4 } & \text { bound } \\
\text { TCNQ }^{\mathrm{x}-} & & \end{array}$ & $1000 \mathrm{~nm}$ & $1.3 \mathrm{ps}(0.92)$ & $16 \mathrm{ps}(0.08)$ & not present \\
\hline
\end{tabular}

${ }^{a}$ Time constant is fixed at the value measured for the PbS QD-only sample.

In order to confirm that the electron and hole are annihilated in one concerted step, we monitored the photoinduced absorption signals of these carriers in the mid-IR. Lian and coworkers have shown that kinetic traces extracted from the mid-IR TA spectrum of PbS QDs between 3 and $4 \mu \mathrm{m}$ have contributions from intraband transitions of both the excitonic electron and hole, whereas traces extracted at $5 \mu \mathrm{m}$ only have contributions from intraband transitions of the hole.[14]We have independently confirmed this finding (see the Supporting Information, Figures S10 and S11).Figure 3B shows kinetic traces extracted from the mid-IR TA spectra, at $4 \mu \mathrm{m}$ and $5 \mu \mathrm{m}$, of a sample of PbS QDs in the absence of TCNQ (orange squares and blue open triangles) and in the presence of 3.3 bound $\mathrm{TCNQ}^{\mathrm{x}}$ - per QD (black triangles and green circles). As expected, the intraband signal does not decay on the picosecond timescale for the sample containing only $\mathrm{PbS} \mathrm{QDs}$ at either wavelength. For the sample of $\mathrm{PbS}$ QDs with 3.3 boundTCNQ ${ }^{\mathrm{x}-}$, we observe a fast decay component with a lifetime of 3.1ps (similar to the 3.3-ps lifetime measured at $1000 \mathrm{~nm})$, and a long-lived shelf $(\tau=2.3 \mu \mathrm{s})$ at both $4 \mu \mathrm{m}$ and $5 \mu \mathrm{m}$. The fact that the kinetic traces at $4 \mu \mathrm{m}$ (where both electron and hole contribute to the signal) and 5 $\mu \mathrm{m}$ (where only the hole contributes to the signal) overlap perfectly further prove that the electron and the hole decay simultaneously in the presence of $\mathrm{TCNQ}^{\mathrm{x}-}$.

The concerted, ultrafast, non-radiative annihilation of the electron and hole of the band-edge exciton of the QD could be due to (i) Auger recombination, (ii) Forster-type energy transfer, or 
(iii) a concerted electron-hole charge transfer (a Dexter-type energy transfer). The singlepicosecond time constants we observe for this process, and the lack of a highly coupled, delocalized charge carrier to accept the energy from the exciton, probably rule out the Auger process. Time constants for Auger recombination are typically 10-400 ps for biexcitons in $\mathrm{PbS}$ QDs, [16-18], and $50 \mathrm{ps}-1.5 \mathrm{~ns}$ for trions in CdSe and CdSe/CdX core/shell QDs.[19-21]. Forster energy transfer is not possible in this system, as there is no overlap between the emission spectrum of the QD and the absorption spectrum of reduced TCNQ, or the complex it makes with sulfur on the surface of the QD (see the Supporting Information, Figure S13). The simultaneous electron-hole transfer we propose as the quenching process is similar to Dexter energy transfer, where instead of the electron and hole being transferred to one localized chromophore, they are transferred to the oxidized sulfur- $\mathrm{TCNQ}^{1-}$ ion pair (or charge-transfer complex, oxidized sulfur-TCNQ ${ }^{\mathrm{x}-}$, in the case of some adsorbed TCNQs). The plot in Figure 3C supports the mechanism of a concerted charge transfer as the PL quenching mechanism. In this figure, we show that the observed rate constant for ultrafast exciton decay $\left(1 / \tau_{1}\right)$ is approximately a linear function of the number of bound $\mathrm{TCNQ}^{\mathrm{x}-}$ per $\mathrm{QD}$, a dependence characteristic of charge transfer in single donor-multi-acceptor systems composed of nanoparticles, [22,23] and molecular redox centers. [24]

In order to support the concerted charge transfer mechanism for PL quenching, and to clarify the roles of both $\mathrm{TCNQ}^{\mathrm{X}-}$ and the surface hole formed upon the spontaneous reduction of TCNQ in this mechanism, we added pre-electrolyzed $\mathrm{TCNQ}^{1-}$ (with tetrabutylammonium, $\mathrm{TBA}^{+}$, counterion) to the QDs rather than allowing the TCNQ to be reduced in situ. When we add 2.5 equivalents of $\mathrm{TBA}^{+} / \mathrm{TCNQ}^{1-}$ to $1.7 \mu \mathrm{MPbS}$ QDs in $\mathrm{CHCl}_{3}$, we observe that $3.4 \%$ of the sample's PL is quenched, whereas $40 \%$ of the sample's PL is quenched when we add this same number of equivalents of TCNQ to the QDs insolution, (see the Supporting Information, Figure S14). It appears, however, that the pre-electrolyzed $\mathrm{TCNQ}^{1-}$ does not quench the PL of the QDs in this experiment because the $\mathrm{TBA}^{+}$stabilizes the $\mathrm{TCNQ}^{1-}$ in solution such that it does not adsorb in high yield to the surfaces of the QDs. The presence of $\mathrm{TCNQ}^{1-}$ in solution, rather than on the QDs, is shown by the higher extinction of $\mathrm{TCNQ}^{1-}$ in the spectrum where it is preelectrolyzed (Figure S14A) vs. when it is formed in situ (Figure S14C).

Furthermore, when we add 3.5 equivalents of neutral TCNQ to $17.8 \mu \mathrm{M} \mathrm{PbS}$ QDs in $\mathrm{CHCl}_{3}$, all of the TCNQ is reduced in situ,Figure 4A (red), and the PL of the sample is quenched by 
$85 \%$ (similar to the $80 \%$ quenching we reported for a separate sample), Figure 4B (red). If we then add salt $\left(\mathrm{TBAPF}_{6}\right)$ to the sample, some of the $\mathrm{TCNQ}^{1-}$ that was reduced on the surfaces of the QDs desorbs, increasing its extinction coefficient, Figure 4A (green) and the PL of the QDs partially recovers, Figure 4B (green). FTIR spectra of $17.8 \mu \mathrm{M}$ PbS QD/TCNQ mixtures, with 12.4 $\mathrm{TCNQ}^{\mathrm{x}-}$ bound per QD, show that, upon addition of $\mathrm{TBAPF}_{6}$, peaks (“2”, $2179 \mathrm{~cm}^{-1}$ ) and (“4”, $2154 \mathrm{~cm}^{-1}$ ) from the spectrum in Figure 1B, corresponding to $\mathrm{TCNQ}^{1-}$ electrostatically bound to the QD surface, are selectively enhanced as this species of reduced TCNQ is released into solution, Figure $4 \mathrm{C}$. This result is reasonable given that $\operatorname{TCNQ}^{\mathrm{x}-}(1<\mathrm{x}<2)$, which corresponds to peaks " 3 " and " 5 " in the spectrum, shares charge with the surface sulfur and therefore cannot desorb as a distinct anion.

The experiments we just described show that $\mathrm{TCNQ}^{1-}-$ specifically $\mathrm{TCNQ}^{1-}$ adsorbed to the surface of the QD - is at least partially responsible for the PL quenching of the QDs, but do not reveal the role of the surface hole in the PL quenching, and do not reveal the mechanism by which the quenching occurs. To answer these questions, we added to the QDs ferrocinium $\left(\mathrm{Fc}^{+}\right)$, which is spontaneously reduced to ferrocene upon adsorption to the surfaces of PbS QDs. Alivisatos and co-workers have previously observed a similar reaction, in which decamethylferrocinium is spontaneously reduced by PbSe films.[25]We chose this oxidant because, unlike $\mathrm{TCNQ}^{1-}$, the reduced form, ferrocene, cannot participate in either electron or hole transfer with the QDs - both processes are energetically uphill. With this system, we therefore eliminate the participation of the reduced species in charge-transfer-type quenching of the QDs' PL. 

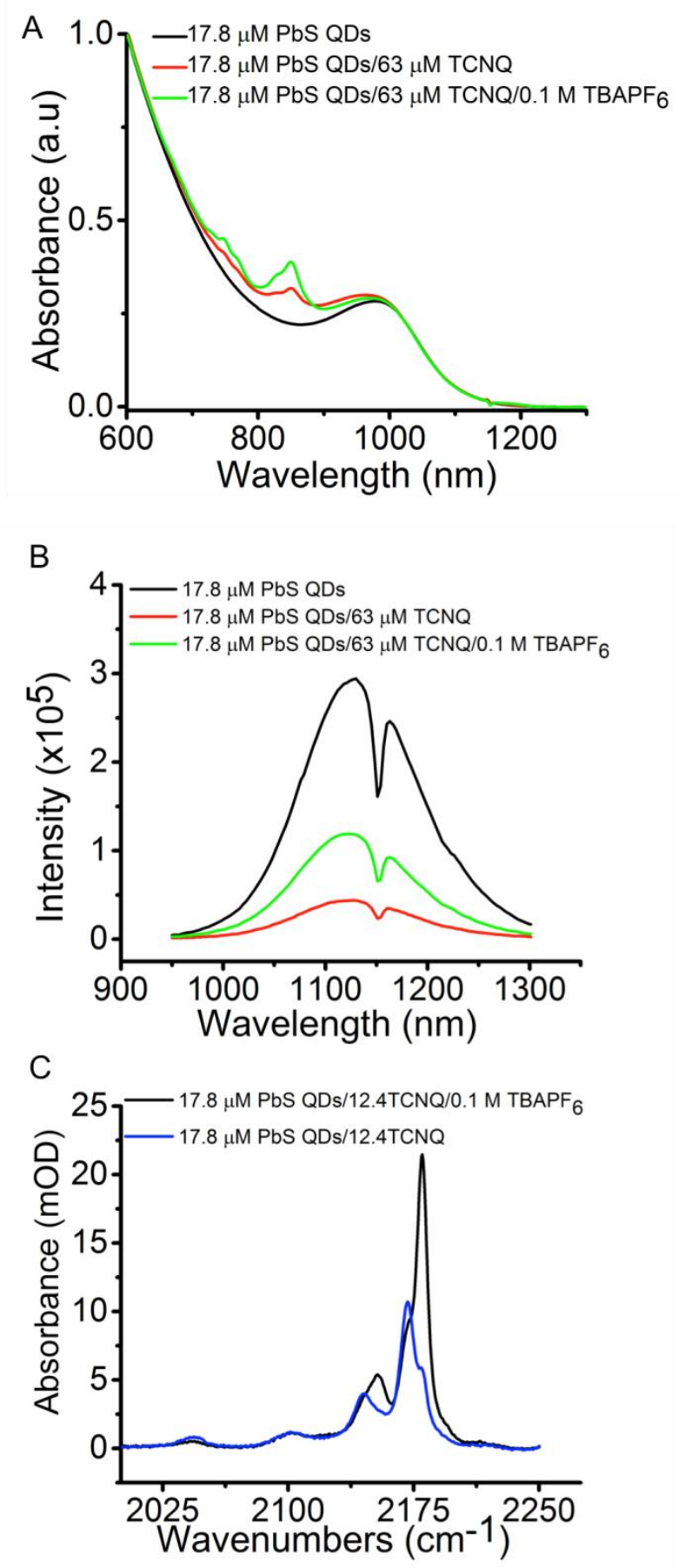

Figure 4.A) Absorption spectra of $17.8 \mu \mathrm{M}$ PbS QDs (black), a mixture of 17.8 $\mu \mathrm{M}$ PbS QDs and $63 \mu \mathrm{M}$ TCNQ (red), and a mixture of $17.8 \mu \mathrm{M}$ PbS QDs, $63 \mu \mathrm{M}$ TCNQ, to which we subsequently added $0.1 \mathrm{M} \mathrm{TBAPF}_{6}$ (green). B) PL spectra of the same set of samples. When we add $0.1 \mathrm{M} \mathrm{TBAPF}_{6}$ to the QD-TCNQ mixture, the QD PL recovers by $25 \%$. The negative feature in the PL spectra is due to reabsorption by a chloroform overtone.C)FTIR spectra of $17.8 \mu \mathrm{M}$ $\mathrm{PbS}$ QDs and 12.4 equivalents of bound $\mathrm{TCNQ}^{\mathrm{x}-}$ before (blue) and after (black) adding $0.1 \mathrm{M}$ TBAPF $_{6}$. The selective enhancement of the peaks at $2179 \mathrm{~cm}^{-1}$ and $2154 \mathrm{~cm}^{-1}$ (peaks " 2 " and "4" from Figure 1B), shows that adding salt desorbs the TCNQ reduced to the 1- state, as opposed to $\mathrm{TCNQ}^{\mathrm{x}-}$, where $1<\mathrm{x}<2$. 
Figure 5A (black) shows the proton NMR spectra of $17.8 \mu \mathrm{M} \mathrm{PbS}$ QDs, of $2 \mathrm{mM} \mathrm{Fc}^{+} / \mathrm{BF}_{4}^{-}$ (green), and of a mixture of $17.8 \mu \mathrm{M} \mathrm{PbS}$ QDs and $50 \mu \mathrm{M} \mathrm{Fc}^{+} / \mathrm{BF}_{4}^{-}$(red), in $\mathrm{CDCl}_{3} \cdot \mathrm{Fc}^{+}$is paramagnetic, and therefore does not display any proton resonance in its NMR spectrum. When $50 \mu \mathrm{M} \mathrm{Fc}{ }^{+} / \mathrm{BF}_{4}^{-}$is added to $\mathrm{PbS}$ QDs, a peak corresponding to $48 \mu \mathrm{M}$ ferroceneappears as a resonance at 4.14 ppm (Figure 5A, inset). Figure5B shows that the PL of the QDs is quenched by only $2 \%$ upon the addition of $\mathrm{Fc}^{+}$and its spontaneous reduction to $\mathrm{Fc}$ (the small amount of quenching is probably due to residual $\mathrm{Fc}^{+}$in solution), despite the formation of a surface hole in this reaction. This result indicates that i) the surface hole does not quench the PL of the QDs on its own, and ii) PL quenching does not occur when the charge transfer pathway is eliminated.

In summary, our data indicate that reduced TCNQ must be surface-adsorbed in order to quench the PL of the QDs, and strongly suggest that this quenching is through charge transfer. The simultaneous electron and hole relaxation that we observe in the near-infrared and midinfrared TA spectra of the samples indicates that both $\mathrm{TCNQ}^{\mathrm{x}-}$ and the surface hole probably participate in a double-charge transfer, Scheme 2.

It is difficult to conclusively say which excitonic carrier recombines with which member of the oxidized sulfur-TCNQ ${ }^{\mathrm{x}-}$ ion pair, because the surface sulfur state, the valence band-edge of the QD, and the TCNQ LUMO are closely spaced in energy. We believe that we do not detect the recombined state because, after the proposed four-carrier charge recombination occurs, the re-reduction of TCNQ to $\mathrm{TCNQ}^{\mathrm{x}-}$ (the thermodynamically favored state) occurs spontaneously and fast enough such that we never observe neutral TCNQ in our transient experiments. The ultrafast PL quenching and re-reduction of TCNQ to TCNQ ${ }^{\mathrm{x}-}$ observed in this study suggest the presence of strong coupling between TCNQ and the surface sulfur. We believe that the covalenttype interactions between TCNQ and the QD observed in the mid-IR (nitrile stretching peaks "3", "5", and "6" in Figure 1B) are evidence for the presence of strong coupling. Spectroscopic signatures of this covalent-type interaction are not clearly discernable in the NIR or visible region of the ground state or TA spectra, probably because they are obscured by the QD spectra, but we have observed them previously for complexes of TCNQ and CdSe QDs.[15] 

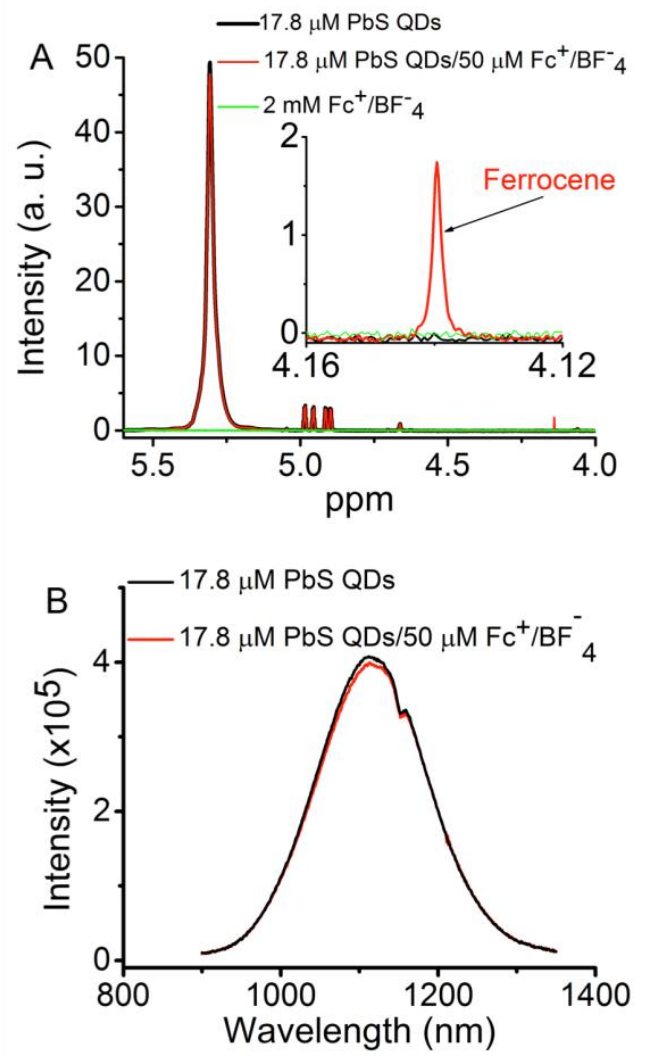

Figure 5.A) Proton NMR spectra of $17.8 \mu \mathrm{M}$ PbS QDs (black), a mixture of $17.8 \mu \mathrm{M}$ PbS QDs and $50 \mu \mathrm{M} \mathrm{Fc}^{+} / \mathrm{BF}_{4}^{-}$(red), and a $2 \mathrm{mM}$ solution of $\mathrm{Fc}^{+} / \mathrm{BF}_{4}^{-}$(green), in $\mathrm{CDCl}_{3}$. The peak centered at $5.3 \mathrm{ppm}$ is the vinyl proton resonance of the native oleate ligand on the surface of the QDs. $\mathrm{Fc}^{+}$does not display any proton resonance in its NMR spectrum because it is a paramagnetic species. Inset: The Fc proton resonance at $4.14 \mathrm{ppm}$ only appears upon mixing $\mathrm{Fc}^{+}$with the QDs. B) PL spectra of the same QD samples. The "dip" observed in the PL spectra is due to reabsorption by a $\mathrm{CDCl}_{3}$ overtone.

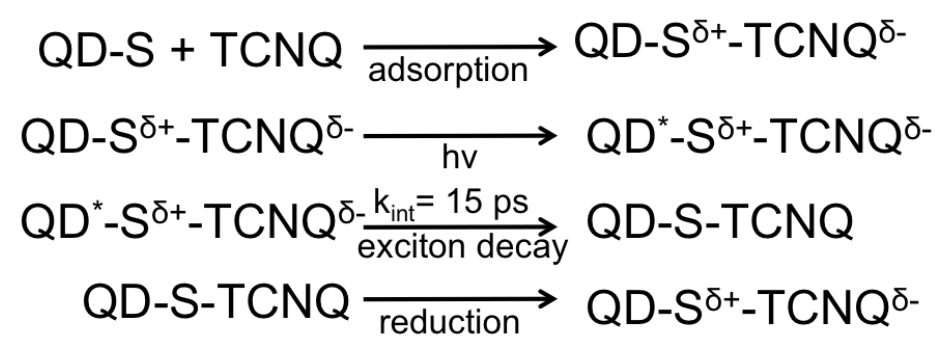

Scheme 2.A sulfur state on the surface of a $\mathrm{PbS}$ QD spontaneously reduces a neutral TCNQ in solution to surface-adsorbed $\mathrm{TCNQ}^{\mathrm{x}-}$ (where $1 \leq \mathrm{x}<2$ ), and becomes oxidized. Selective

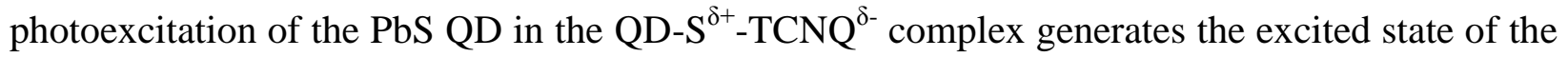
QD. The excited QD decays with an intrinsic lifetime of $15 \mathrm{ps}$, probably through a double-charge transfer process whereby the QD returns to its ground state, and the sulfur and TCNQ are 
restored to their original (pre-adsorbed) oxidation states. This excited state decay process is followed by the fast, spontaneous re-reduction of TCNQ by the surface sulfur.

\section{CONCLUSIONS}

We have shown using ground state absorption spectroscopy that $\mathrm{PbS}$ QDs $(\mathrm{R}=1.8 \mathrm{~nm})$ spontaneously reduce up to 17 TCNQ molecules per QD in solution to form multi-plexed charge transfer complexes. Within these complexes, some adsorbed TCNQ molecules undergo a oneelectron reduction to form $\mathrm{TCNQ}^{1-}$, and some share electron density with their chalcogenide surface site to form $\mathrm{TCNQ}^{\mathrm{x}-}, 1<\mathrm{x}<2$. The presence of surface-adsorbed, reduced TCNQ species quenches the PL of the QDs. Using a combination of time-resolved near-IR and mid-IR transient absorption spectroscopy, we determined that the PL quenching occurs through an ultrafast, nonradiative, simultaneous decay of the excitonic electron and hole. The rate constant for this decay increases linearly with the number of adsorbed $\mathrm{TCNQ}^{\mathrm{x}-}$ per $\mathrm{QD}$, and has an intrinsic time constant of 15 ps. Removal of adsorbed $\mathrm{TCNQ}^{1-}$ from the surface of the QD through addition of a counterion (tetrabutylammonium) causes the PL to recover. Addition of a different spontaneous oxidant, ferrocinium, to the QDs also produces a localized hole on the QD surface, but does not quench the QDs' PL, probably because the reduced product, ferrocene, is not redox active with respect to the QD exciton. These data allow us to propose that the PL quenching and ultrafast exciton decay upon adsorption of TCNQ and spontaneous reduction to TCNQ ${ }^{\mathrm{x}-}$ occurs through a four-carrier concerted charge recombination, where the excitonic electron and hole simultaneously recombine with the oxidized sulfur-TCNQ ${ }^{\mathrm{x}-}$ ion pair on the surface of the QD. We do not directly detect the product of this recombination because, we believe, the system spontaneously reverts to the oxidized sulfur- $\mathrm{TCNQ}^{\mathrm{x}-}$ state too fast for us to measure the neutral intermediate.

The observation of ultrafast exciton recombination in the presence of reduced TCNQ indicates that, if one is to use a QD as a sensitizer and initial electron or hole donor within a sequential photoinduced multi-charge transfer process (such as those necessary for many catalytic reactions), the presence of a surface-localized pre-formed ion pair potentially serves as a recombination site for the QD exciton, with charge recombination rates that can outcompete subsequent charge transfer steps. It may therefore be difficult to accumulate electrons or holes on 
the surface of a QD, in which case either charge transfer to the catalytic substrate or scavenging of the second carrier will have to be efficient for the QD to perform multi-electron reactions.

\section{ACKNOWLEDGEMENTS}

This research was supported as partof the Argonne-Northwestern Solar Energy Research (ANSER) Center, an Energy Frontier Research Center funded by the U.S. Department of Energy (DOE), Office of Science, Basic Energy Sciences (BES), via Grant DE-SC0001059, and by the National Science Foundation through a Graduate Research Fellowship to K.E. (DGE-1324585). Any opinion, findings, and conclusions or recommendations expressed in this material are those of the authors(s) and do not necessarily reflect the views of the National Science Foundation.FTIR was performed in the Keck-II facility of NUANCE Center at Northwestern University. The NUANCE Center is supported by the International Institute for Nanotechnology, MRSEC (NSF DMR-1121262), the Keck Foundation, the State of Illinois, and Northwestern University. The authors thank Michael Mattei for his help in performing bulk electrolysis experiments, and Dr. Christopher Thompson for helpful discussions.

\section{References}

[1] K.E. Knowles, M. Malicki, R. Parameswaran, L.C. Cass, E.A. Weiss, J. Am. Chem. Soc. 135 (2013) 7264.

[2] D.L. Dexter, J. Chem. Phys. 21 (1953) 836.

[3] N.J. Thompson, M.W.B. Wilson, D.N. Congreve, P.R. Brown, J.M. Scherer, Thomas S. Bischof, M. Wu, N. Geva, M. Welborn, T.V. Voorhis, V. Bulović, M.G. Bawendi, Marc A. Baldo, Nat Mater 13 (2014) 1039.

[4] A. Khetubol, S. Van Snick, A. Hassinen, E. Fron, Y. Firdaus, L. Pandey, C.C. David, K. Duerinckx, W. Dehaen, Z. Hens, M. Van der Auweraer, J. Appl. Phys. 113 (2013) 083507.

[5] S.E. Miller, A.S. Lukas, E. Marsh, P. Bushard, M.R. Wasielewski, J. Am. Chem. Soc. 122 (2000) 7802.

[6] M.G. Bawendi, M.L. Steigerwald, L.E. Brus, Annu. Rev. Phys. Chem. 41 (1990) 477.

[7] J.B. Sambur, T. Novet, B.A. Parkinson, Science 330 (2010) 63.

[8] B.L. Wehrenberg, P. Guyot-Sionnest, J. Am. Chem. Soc. 125 (2003) 7806.

[9] M.A. Hines, G.D. Scholes, Adv. Mater. 15 (2003) 1844.

[10] E.A. McArthur, A.J. Morris-Cohen, K.E. Knowles, E.A. Weiss, J. Phys. Chem. B 114 (2010) 14514.

[11] I. Moreels, K. Lambert, D. Smeets, D. De Muynck, T. Nollet, J.C. Martins, F. Vanhaecke, A. Vantomme, C. Delerue, G. Allan, Z. Hens, ACS Nano 3 (2009) 3023. 
[12] S.E. Bell, J.S. Field, R.J. Haines, M. Moscherosch, W. Matheis, W. Kaim, Inorg. Chem. 31 (1992) 3269.

[13] L.R. Melby, R.J. Harder, W.R. Hertler, W. Mahler, R.E. Benson, W.E. Mochel, J. Am. Chem. Soc. 84 (1962) 3374.

[14] Y. Yang, W. Rodríguez-Córdoba, T. Lian, J. Am. Chem. Soc. 133 (2011) 9246.

[15] L.C. Cass, N.K. Swenson, E.A. Weiss, J. Phys. Chem. C 118 (2014) 18263.

[16] Y. Yang, W. Rodríguez-Córdoba, T. Lian, Nano Lett. 12 (2012) 4235.

[17] J.T. Stewart, L.A. Padilha, M.M. Qazilbash, J.M. Pietryga, A.G. Midgett, J.M. Luther, M.C. Beard, A.J. Nozik, V.I. Klimov, Nano Lett. 12 (2012) 622.

[18] F. Gesuele, M.Y. Sfeir, W.K. Koh, C.B. Murray, T.F. Heinz, C.W. Wong, Nano Lett. 12 (2012) 2658.

[19] A.W. Cohn, J.D. Rinehart, A.M. Schimpf, A.L. Weaver, D.R. Gamelin, Nano Lett. 14 (2014) 353.

[20] K. Gong, D.F. Kelley, J. Phys. Chem. C 119 (2015) 9637.

[21] H.M. Zhu, N.H. Song, T.Q. Lian, J. Am. Chem. Soc. 135 (2013) 11461.

[22] M. Tachiya, J. Chem. Phys 76 (1982) 340.

[23] A.J. Morris-Cohen, M.T. Frederick, L.C. Cass, E.A. Weiss, J. Am. Chem. Soc. 133 (2011) 10146.

[24] M.J. Ahrens, R.F. Kelley, Z.E.X. Dance, M.R. Wasielewski, PCCP 9 (2007) 1469.

[25] J.H. Engel, Y. Surendranath, A.P. Alivisatos, J. Am. Chem. Soc. 134 (2012) 13200. 


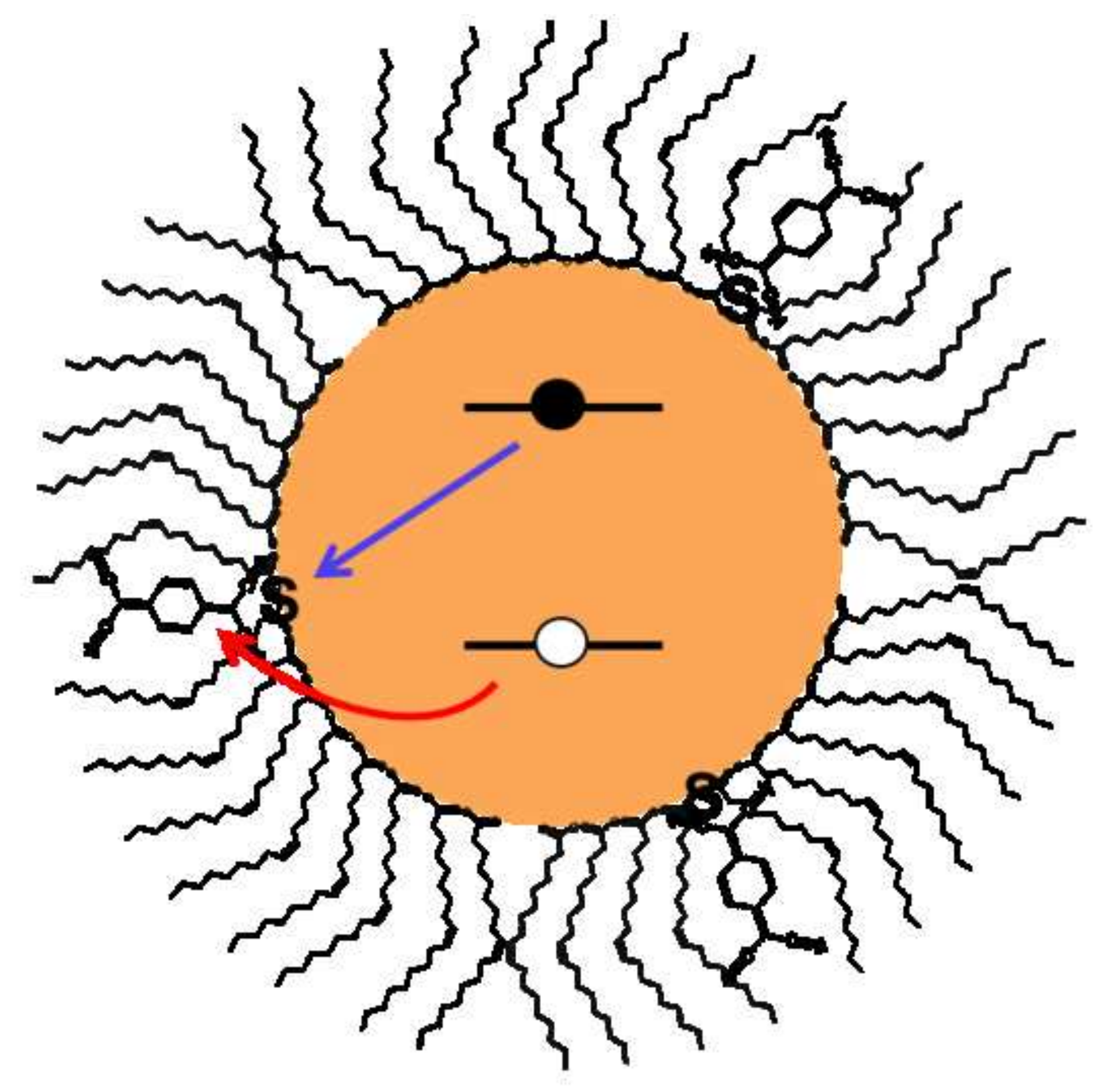

